\title{
Waiting time phenomena of the Hele-Shaw and the Stefan problem
}

\author{
Sunhi Choi and Inwon Kim \\ Department of Mathematics, MIT
}

May 25, 2005

\begin{abstract}
In this paper we investigate the waiting time phenomena for the one-phase Hele-Shaw and Stefan problems. We identify a general criterion on the growth rate of the initial data for the Hele-Shaw problem which determines the occurrence of a waiting time. For the Stefan problem we show that the waiting time phenomena depends on the balance between the initial data and the geometry of the initial positive phase.
\end{abstract}

\section{Introduction}

Let us consider a compact set $K \subset \mathbb{R}^{n}$ with smooth boundary $\partial K$. Suppose that a bounded domain $\Omega$ contains $K$ and let $\Omega_{0}=\Omega-K$ and $\Gamma_{0}=\partial \Omega$ (Figure 1). Note that $\partial \Omega_{0}=\Gamma_{0} \cup \partial K$.

Let $u_{0}$ be the harmonic function in $\Omega_{0}$ with smooth fixed boundary data $u_{0}=f>0$ on $K$ and $u_{0}=0$ on $\Gamma_{0}$. The classical Hele-Shaw problem, in

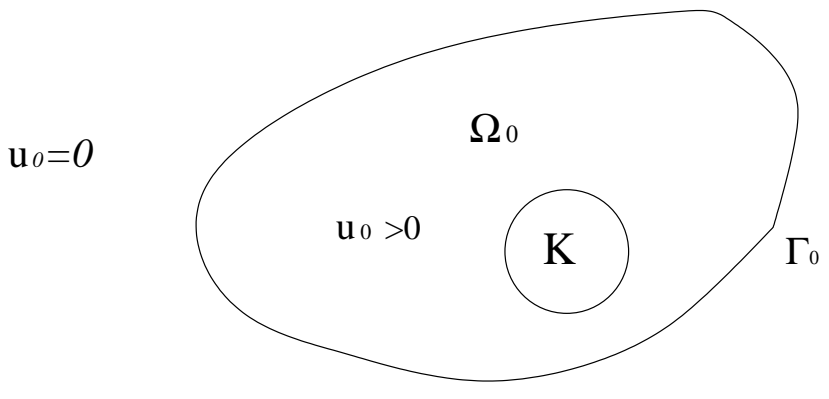

Figure 1. 
$n=2$, models an incompressible viscous fluid which occupies part of the space between two parallel, narrowly placed plates ([ST], [EJ].) In this case $u_{0}$ denotes the initial pressure of the fluid and $f$ denotes the rate of injection from $K$ into $\Omega_{0}$. Assuming that there is no surface tension, the pressure of the fluid $u(x, t)$ satisfies

$$
\begin{cases}-\Delta u=0 & \text { in }\{u>0\} \cap Q, \\ u_{t}-|D u|^{2}=0 & \text { on } \partial\{u>0\} \cap Q, \\ u(x, 0)=u_{0}(x) ; & u(x, t)=f \text { for } x \in \partial K .\end{cases}
$$

where $Q=\left(\mathbb{R}^{n}-K\right) \times(0, \infty)$. Observe that the initial data $u_{0}$ in (HS) is determined by the initial domain $\Omega_{0}$.

We define

$$
\Gamma(u)=\partial\{u>0\}, \Gamma_{t}(u)=\partial\{u(\cdot, t)>0\}-\partial K
$$

respectively the free boundary of $u$ and the free boundary of $u$ at time $t$. We also define

$$
\Omega(u)=\{u>0\}, \Omega_{t}(u)=\{u(\cdot, t)>0\}
$$

respectively the positive phase of $u$ and the positive phase of $u$ at time $t$. When it is obvious from the context we will omit $u$ in the notation of $\Omega_{t}(u)$ and $\Gamma_{t}(u)$.

Note that if $u$ is smooth up to the free boundary, then the free boundary moves with normal velocity $V=u_{t} /|D u|$, and hence the second equation in (HS) implies that $V=|D u|$. Also note that $u$ is determined by $\Omega_{0}$ and $f$.

The classical Stefan problem accounts for phase transitions between solid and fluid states, such as the melting of ice in contact with water ([Ru],[Me].) Here we assume that the temperature varies only in fluid and the temperature of the solid is maintained at $0^{0} C$. Then the temperature distribution of the fluid $u(x, t)$ with nonnegative initial data $u_{0}$ satisfies

$$
\begin{cases}u_{t}-\Delta u=0 & \text { in }\{u>0\} \\ u_{t}-|D u|^{2}=0 & \text { on } \partial\{u>0\} \\ u(x, 0)=u_{0}(x) & \end{cases}
$$




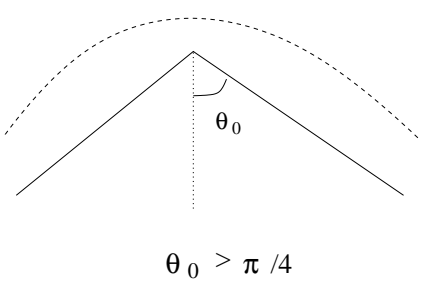

Free boundary smoothes out instantly

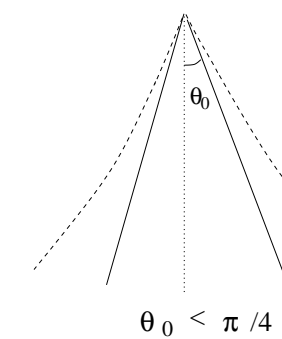

There is a waiting time at the vertex

Figure 2.

We use the same notation for the free boundary and the positive phase of $u$. (Here the free boundary of $u$ is given as $\Gamma_{t}(u)=\partial\{u(\cdot, t)>0\}$.) Note that in both problems (HS)-(ST) the free boundary expands in time.

We say that a solution of (HS) or (ST) has a (initial) waiting time $t_{0}>0$ at $P \in \mathbb{R}^{n}$ if $P \in \Gamma_{0}$ and $P \in \Gamma_{t}(u)$ for $0<t<t_{0}$.

For $n=2[\mathrm{KLV}]$ studied the behavior of self-similar solutions of (HS) with $\Gamma_{0}$ as 'wedges' of the form

$$
\left\{(r, \theta):|\theta|=\theta_{0}\right\}
$$

It is proven here that if $\theta_{0} \geq \pi / 4$ then the free boundary strictly expands and smoothes out, and if $\theta_{0}<\pi / 4$ then there is a waiting time at the vertex of the wedge (see Figure 2.)

In fact parallel result holds (see [JeKi]) for radially symmetric solutions in $\mathbb{R}^{n}, n>2$ with the threshold angle $\theta_{0}=\theta_{n}$, with which the initial data has a quadratic decay rate at the vertex, i.e.,

$$
u_{0}(r, 0) \sim r^{2} .
$$

For solutions of (HS) with Lipschitz initial domain $\Omega$ in $\mathbb{R}^{n}, n \geq 2$ it is recently proved in [CJK] that if the Lipschitz constant of the domain is smaller than a dimensional constant $a_{n}$, then the free boundary immediately smoothes out, that is $\Gamma(u)$ becomes analytic in space and time for a small amount of positive time. In particular for $n=2$ we have $a_{2}=1=\cot \pi / 4$, which corresponds to the threshold angle $\theta_{0}=\pi / 4$ in the analysis of [KLV]. For $n>2$ we have $a_{n}$ smaller than $\cot \theta_{n}$ due to technical reasons. For the two- dimensional self-similar solutions of (ST), with $\Gamma_{0}$ given as in (0.1) and with $u_{0}=1$ in $\Omega_{0}(u)$, [K] shows that parallel results holds with a threshold angle $\theta_{0}=\pi / 6$. 
An open and interesting question, positively answered from above results, is whether there is a dichotomy on the free boundary behavior of (HS) and (ST) near $t=0$, that is whether it is always one of the two cases: either the free boundary immediately smooths out, or there is a waiting time at a point on the initial free boundary. (We mention that the regularity of the free boundary may not last for all time due to the collision of free boundary parts.)

In this paper we ask which information on the initial data determines the occurrence of the waiting time at a given point on the initial free boundary for both problems (HS) and (ST).

Our goal in this paper is

(1) to extend the results of [KLV] on self-similar solutions in $\mathbb{R}^{2}$ to solutions with Non-Tangentially Accessible initial domains in $\mathbb{R}^{n}$ and

(2) to investigate the waiting time phenomena for solutions of (ST) with Lipschitz initial domain in $\mathbb{R}^{n}$.

In regards to (1) the main idea for the proof is that the occrrence of waiting time depends on the 'pushing force' at the vertex, which is generated in the neighboring regions and accumulated with respect to the distance to the vertex. (See for example (1.3).)

In regards to (2) we will prove that the waiting time phenomena for (ST) depends on the balance between the initial heat $u_{0}$ and the geometry of the initial positive phase $\Omega_{0}$ of $u$ (see Theorem 1.6- 1.7). Roughly speaking the waiting time occurs when the initial heat $u_{0}$ can change into the latent heat (harmonic function) without changing the geometry of the domain too much. It is proven in $[\mathrm{K}]$ that with a strong, discontinuous initial heat $u_{0}=1 \mathrm{in}$ the positive phase and with $\Gamma_{0}$ given as in (0.1), we require the half-angle of the wedge $\theta_{0}>\pi / 6$ for the free boundary to immediately expand and smooth out. With harmonic initial data, $\Gamma_{0}$ needs to be flatter to smooth out: we require that $\theta_{0}>\pi / 4$. This is plausible since the initial heat for the first case is much stronger than in the second case, and thus melts the ice more easily.

In Theorem 1.7 we will show that indeed the initial heat needs to be much stronger than the harmonic initial data to make a difference in the waiting time phenomena. More precisely we prove that if the initial data has a degree of regularity depending on the geometry of the initial positive phase, than the occurrence of the waiting time for (ST) will coincide with the case of harmonic initial data. This is because if $u_{0}$ is regular enough 
then the harmonic measure associated with the evolving positive phase does not change too much while the initial heat changes into 'almost' latent heat. (For further discussion see section 5.) In particular when $\Gamma_{0}$ is given as in (0.1) it follows from Theorem 1.6-1.7 that any Hölder continuous initial data $u_{0}$ will generate the same waiting time phenomena with the case of harmonic initial data, that is there is a waiting time if $\theta_{0}<\pi / 4$ and no waiting time if $\theta_{0}>\pi / 4$.

In contrast, in the case of the porous medium equation

$$
(P M E)_{m} \quad u_{t}-m u \Delta u-|D u|^{2}=0, u \geq 0,
$$

the waiting time occurrence is solely determined by the decay rate of the initial data $u_{0}$ (see $[\mathrm{A}],[\mathrm{AC}]$ ), independently of the geometry of the initial positive phase.

For our investigation we use the notion of viscosity solutions, which has been recently introduced in [Ki1]. An important property of viscosity solutions which is frequently used in this paper is the comparison principle (see Theorem 2.7), which enables us to compare our solutions with barriers that we construct in various settings. The main tools we use in the paper include

(1) comparison principle of solutions for (HS) and (ST),

(2) estimates on harmonic measures in Lipschitz and NTA domains, and

(3) properties of caloric functions in Lipschitz domains.

\section{Statements of the main results}

In this section we summarize the results of this paper. Here we use viscosity solutions (see section 2 for definitions and properties) introduced in [Ki1] as our notion of solutions.

\subsection{Hele-Shaw problem}

For $x \in \mathbb{R}^{n}$ we denote $B_{r}(x):=\left\{y \in \mathbb{R}^{n}:|y-x|<r\right\}$. we let $\Omega$ be a bounded domain in $\mathbb{R}^{n}$ such that $0 \in \partial \Omega$ and let $K \subset B_{1 / 2}\left(-e_{n}\right) \subset \Omega$. 


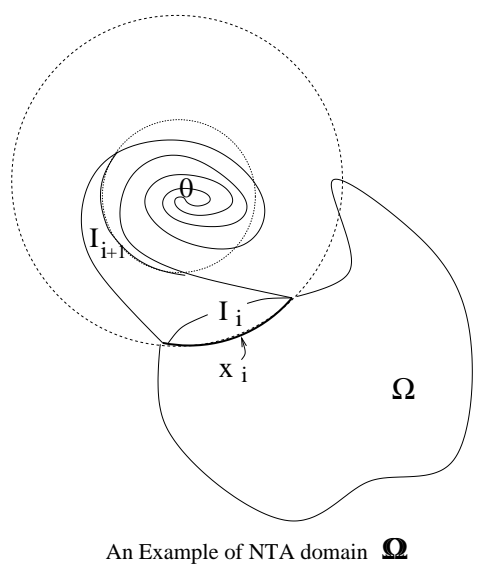

Figure 3.

Definition 1.1. $\Omega$ is called non-tangentially accessible (NTA) when there exists a constant $M>1$ such that:

1. Corkscrew condition. For any $\zeta \in \partial \Omega, r<1$ there exists $y=y(r, \zeta) \in \Omega$ such that $r / M<|y-\zeta|<r$ and $B_{r / M}(y) \subset \Omega$.

2. $\Omega^{c}$ satisfies corkscrew condition.

3. Harnack Chain Condition. Let $\epsilon>0$ and let $x_{1}, x_{2} \in \Omega \cap B_{r / 4}(\zeta)$ for some $\zeta \in \partial \Omega$ and $r<1$. If $\operatorname{dist}\left(x_{j}, \partial \Omega\right)>\epsilon$ and $\left|x_{1}-x_{2}\right|<2^{k} \epsilon$, then there exists a Harnack chain from $x_{1}$ to $x_{2}$ of length $M k$ such that the diameter of each ball is bounded below by $M^{-1} \min \left\{\operatorname{dist}\left(x_{1}, \partial \Omega\right), \operatorname{dist}\left(x_{2}, \partial \Omega\right)\right\}$.

$M$ is called the NTA constant.

NTA domains include Lipschitz domains and (slowly rotating) spiral domains (see Figure 3.)

Definition 1.2. Let $\Omega$ be an NTA domain with NTA constant $M$ and let $0 \in \partial \Omega,-e_{n} \in \Omega$. We denote by $I_{i}$ the component of $\Omega \cap \partial B_{2^{-i}}(0)$ which separates 0 from $-e_{n}$, i.e. 0 and $-e_{n}$ are contained in the different components of $\Omega-I_{i}$ (see Figure 3). We also denote by $x_{i}$ a point on $I_{i}$ such that $B_{2^{-i} / M^{2}}\left(x_{i}\right) \subset \Omega$.

Note that by (i) and (iii) of the NTA conditions there exists such $x_{k} \in I_{k}$.

Throughout Section 1.1 we assume that $\Omega$ is NTA with $\left\{x_{i}\right\}_{i}$ as given in Definition 1.1 and $u$ is the viscosity solution of (HS) with $\Omega_{0}=\Omega-K$, 
$0 \in \Gamma_{0}$. In the next two theorems we extend the result of $[\mathrm{KLV}]$ and show that if $\Omega_{0}$ is NTA then the threshold decay rate of $u_{0}$ for the waiting time phenomena of (HS) is always quadratic.

Theorem 1.3. If there exists $\epsilon>0$ such that $\left|u_{0}\left(x_{i}\right)\right| \leq\left|x_{i}\right|^{2+\epsilon}$ for $i=$ $1,2, \ldots$, then there exists a waiting time $t_{0}>0$ for $u(x, t)$ at 0 . In particular, $t_{0} \geq C \epsilon^{1 / \beta_{n}}$ where $C$ depends on the NTA constant and $\beta_{n}$ is a dimensional constant given in Lemma 3.2.

Remark There are initial (non-NTA) domains such that $\left|u_{0}(x)\right| \leq|x|^{2+\epsilon}$ but $u$ has no waiting time. For a given angle $\theta_{0}$ let us define

$$
\Omega=B_{1}(0) \cap\left\{x_{n}<0\right\}-\cup_{i \geq 1}\left(\partial B_{2^{-i}}(0)-W\left(\theta_{0},-e_{n}\right)\right)
$$

where

$$
W\left(\theta_{0},-e_{n}\right)=\left\{x \in \mathbb{R}^{n}:<x,-e_{n}>\geq|x| \cos \theta_{0}\right\} .
$$

We choose $\theta_{0}<\theta_{n}$, where $\theta_{n}$ is a dimensional constant such that if $\theta_{0}=\theta_{n}$ then the harmonic function associated with $\Omega$ has a quadratic decay at $x=0$. Then for this range of $\theta_{0}$ we have $\left|u_{0}(x)\right| \leq|x|^{2+\epsilon}$ for some $\epsilon>0$.

Let $\Omega_{0}=\Omega-B_{1 / 8}\left(-\frac{3}{4} e_{n}\right)$, then $u$ has no waiting time at $0 \in \Gamma_{0}$ since

$$
B_{1}(0) \cap\left\{x_{n}<0\right\} \subset \Omega_{t} \text { for any } t>0 .
$$

Theorem 1.4. The following statements are true:

(a) If there exists a sequence $n_{j} \rightarrow \infty$ such that $u\left(x_{n_{j}}\right) \geq\left|x_{n_{j}}\right|^{2-\epsilon_{n_{j}}}$, then $u$ has no waiting time.

(b) If $u\left(x_{i}, 0\right)$ is comparable to $\left|x_{i}\right|^{2}$ for $i=1,2, \ldots$, then $u$ has no waiting time.

We next consider the borderline case where $u_{0}$ has quadratic decay at 0 with logarithmic perturbations. In $[\mathrm{KLV}]$ it is proved that if $\Gamma_{0}$ is given as a 'curved wedge'

$\left\{(r, \theta):|\theta| \sim \pi / 4\left(1-\frac{\alpha}{2|\ln (r)|}\right)\right\}$ near 0 so that

$$
u_{0}(z) \sim \operatorname{Re}\left\{\frac{z^{2}}{(-\ln z)^{\alpha}}\right\}_{+} \text {as } z \rightarrow 0,
$$

then there is a waiting time at the vertex if $\alpha>1$ and there is no waiting time if $\alpha<1$. Next theorem extends this result of [KLV]. 
Theorem 1.5. Assume that

(1.2) There exists $m>0$ such that for every $\zeta \in \Gamma_{0}$ there exist balls $B_{1}$ and $B_{2}$ contained in $\Omega_{0}$ and $\mathbb{R}^{n}-\Omega_{0}$ such that $\operatorname{rad}\left(B_{1}\right)=\operatorname{rad}\left(B_{2}\right)=m|\zeta|$ and $\zeta=\partial B_{1} \cap \partial B_{2}$.

Then the following statements hold:

(a) If $u_{0}\left(x_{i}\right) \leq\left|x_{i}\right|^{2}\left(\frac{1}{i}\right)^{\alpha}$ for $\alpha>1$ and $i=1,2, \ldots$, then $u$ has a waiting time.

(b) If $u_{0}\left(x_{i}\right) \geq\left|x_{i}\right|^{2}\left(\frac{1}{i}\right)^{\alpha}$ for $\alpha<1$ and $i=1,2, \ldots$, then $u$ has no waiting time.

\section{Remark}

1. The case $u_{0}(x) \sim\left|x_{i}\right|^{2}(i)^{-\alpha}$ arises, for example, when $\Omega_{0}$ is given as in (1.1) with $n=2$. Roughly speaking these are the cases when $\Omega_{0}$ is a logarithmic perturbation of a domain where the associated harmonic function decays exactly quadratically at zero.

2. (1.2), a $C^{2}$-bound for $\Gamma_{0}$ scaled with respect to the distance to the vertex, is necessary for technical reasons in our proof. It is not clear whether the statement holds without (1.2).

Theorem 1.5 (a) is a special case of the following general statement. Roughly speaking $a_{k}$ corresponds to the 'vertex-pushing force' generated outside of the $2^{-k}$-neghborhood of the vertex.

Proposition 1.6. Let $\Omega$ be NTA with (1.2). Let $a_{0}=1$ and

$$
a_{k}=\Pi_{i=0}^{k-1}\left(1+a_{i}\right) 2^{2 k} u_{0}\left(x_{i}\right) .
$$

If $\lim \sup a_{k}<\infty$, then there exists a waiting time.

\subsection{Stefan problem}

Throughout section 1.2 we denote $u$ the viscosity solution of (ST) with initial data $u_{0}(x)$ such that $\left\{u_{0}(x)>0\right\}=\Omega_{0}$ is a Lipchitz domain in $\mathbb{R}^{n}$. Let $K$ be a compact set in $\Omega$ with a smooth boundary and let $h_{0}(x)$ be the harmonic function in $\Omega_{0}-K$ with boundary data 0 on $\Gamma_{0}=\partial \Omega_{0}$ and 1 on $\partial K$. After a rotation and translation, we may assume that $0 \in \Gamma_{0}$ and $\Gamma_{0}=\left\{x=\left(x^{\prime}, x_{n}\right): x_{n}=f\left(x^{\prime}\right)\right\}$ with a Lipschitz function $f$ in a small neighborhood of 0 with $f(0)=0$. In this section we denote $C$ as a positive constant depending only on the Lipschitz constant of $f$ and dimension $n$. Let positive constants $\alpha_{1}<\alpha_{2}$ and $C$ satisfy 


$$
\frac{1}{C} r^{\alpha_{2}} \leq h_{0}\left(-r e_{n}\right) \leq C r^{\alpha_{1}}
$$

for sufficiently small $r>0$. We call $\alpha_{1}$ and $\alpha_{2}$ respectively the minimal and maximal decay rate of $h_{0}$ at 0 .

Theorem 1.7. If the maximal decay rate of $h_{0}(x)$ at 0 is less than 2 then $u$ has no waiting time at 0 .

Theorem 1.8. Let $u(x, 0) \in C^{\delta}\left(\bar{\Omega}_{0}\right)$ and let $\alpha_{1} \leq \alpha_{2}$ to be the minimal and maximal decay rate of $h_{0}$ at 0 . If $\alpha_{1}>2$ and $\alpha_{2}-\alpha_{1}<\delta / 2$ then $u$ has a waiting time at the origin.

Remark $u$ may not have a waiting time if $\alpha_{2}-\alpha_{1} \geq \delta / 2$, in particular if $u_{0}$ is discontinuous. A good example is the one studied in [K], where $\Omega_{0}$ is given as in (0.1) with $n=2$ (therefore $\alpha_{2}=\alpha_{1}$ ) and $u_{0}$ is the characteristic function of $\Omega_{0}$. In this case there is no waiting time if the wedge angle $\theta_{0}$ is between $\pi / 4$ and $\pi / 6$ even though the decay rate of $h_{0}$ satisfies $\alpha_{1}=\alpha_{2}>2$. In section 5 we construct another example where $\Omega_{0}$ is an oscillatory domain and $u_{0} \in C^{\delta}$ but $\alpha_{2}-\alpha_{1}>\delta / 2$.

\section{Viscosity solutions}

In this section we introduce the notion of viscosity solutions for (HS) and (ST) which we will use in this paper. Roughly speaking, viscosity sub and supersolutions are defined by comparison with local (smooth) super and subsolutions. In particular classical solutions of either problems are also viscosity sub and supersolutions.

Definition 2.1. We say that a pair of functions $u_{0}, v_{0}: \bar{D} \rightarrow[0, \infty)$ are (strictly) separated (denoted by $u_{0} \prec v_{0}$ ) in $D \subset \mathbb{R}^{n}$ if

(i) the support of $u_{0}, \operatorname{supp}\left(u_{0}\right)=\overline{\left\{u_{0}>0\right\}}$ restricted in $\bar{D}$ is compact and

(ii) in $\operatorname{supp}\left(u_{0}\right) \cap \bar{D}$ the functions are strictly ordered:

$$
u_{0}(x)<v_{0}(x)
$$

For a nonnegative real valued function $u(x, t)$ defined in a cylindrical domain $D \times(a, b)$, we define 


$$
\begin{aligned}
& \Omega(u)=\{(x, t): u(x, t)>0\}, \quad \Omega_{t}(u)=\{x: u(x, t)>0\}, \\
& \Gamma(u)=\partial\{(x, t): u(x, t)=0\}, \quad \Gamma_{t}(u)=\partial\{x: u(x, t)=0\} .
\end{aligned}
$$

Let $Q=\left(\mathbb{R}^{n}-K\right) \times(0, \infty)$ and let $\Sigma$ be a cylindrical domain $D \times(a, b) \subset$ $\mathbb{R}^{n} \times \mathbb{R}$, where $D$ is an open subset of $\mathbb{R}^{n}$. The following definitions are introduced in [Ki1].

Definition 2.2. A nonnegative upper semicontinuous function u defined in $\Sigma$ is a viscosity subsolution of $(H S)$ if

(a) for each $a<T<b$ the set $\overline{\Omega(u)} \cap\{t \leq T\}$ is bounded; and

(b) for every $\phi \in C^{2,1}(\Sigma)$ such that $u-\phi$ has a local maximum in $\overline{\Omega(u)} \cap$ $\left\{t \leq t_{0}\right\} \cap \Sigma$ at $\left(x_{0}, t_{0}\right)$,

(i) $-\Delta \phi\left(x_{0}, t_{0}\right) \leq 0 \quad$ if $u\left(x_{0}, t_{0}\right)>0$.

(ii) $\quad\left(\phi_{t}-|D \phi|^{2}\right)\left(x_{0}, t_{0}\right) \leq 0 \quad$ if $\left(x_{0}, t_{0}\right) \in \Gamma(u)$ if $-\Delta \phi\left(x_{0}, t_{0}\right)>0$.

Note that because $u$ is only upper lowercontinuous there may be points of $\Gamma(u)$ at which $u$ is positive.

Definition 2.3. A nonnegative lower semicontinuous function $v$ defined in $\Sigma$ is a viscosity supersolution of $(H S)$ if for every $\phi \in C^{2,1}(\Sigma)$ such that $v-\phi$ has a local minimum in $\Sigma \cap\left\{t \leq t_{0}\right\}$ at $\left(x_{0}, t_{0}\right)$,

$$
\begin{gathered}
\text { (i) } \quad-\Delta \phi\left(x_{0}, t_{0}\right) \geq 0 \quad \text { if } v\left(x_{0}, t_{0}\right)>0, \\
\text { (ii) If }\left(x_{0}, t_{0}\right) \in \Gamma(v),|D \phi|\left(x_{0}, t_{0}\right) \neq 0 \text { and } \\
-\Delta \varphi\left(x_{0}, t_{0}\right)<0,
\end{gathered}
$$

then

$$
\left(\phi_{t}-|D \phi|^{2}\right)\left(x_{0}, t_{0}\right) \geq 0 .
$$

Definition 2.4. $u$ is a viscosity subsolution of (HS) with initial data $u_{0}$ and fixed boundary data $f>0$ if

(a) $u$ is a viscosity subsolution in $\bar{Q}$,

(b) $u=u_{0}$ at $t=0 ; u \leq f$ on $\partial K$; 
(c) $\overline{\Omega(u)} \cap\{t=0\}=\overline{\Omega\left(u_{0}\right)}$;

Definition 2.5. $u$ is a viscosity supersolution of (HS) with initial data $u_{0}$ and fixed boundary data $f$ if $u$ is a viscosity supersolution in $\bar{Q}$ with $u=u_{0}$ at $t=0$ and $u \geq f$ on $\partial K$.

For a nonnegative real valued function $u(x, t)$ defined in a cylindrical domain $D \times(a, b)$,

$$
u^{*}(x, t)=\limsup _{(\xi, s) \in D \times(a, b) \rightarrow(x, t)} u(\xi, s) .
$$

Definition 2.6. $u$ is a viscosity solution of (HS) (with boundary data $u_{0}$ and $f$ ) if $u$ is a viscosity supersolution and $u^{*}$ is a viscosity subsolution of (HS) (with boundary data $u_{0}$ and $f$.)

Viscosity solutions of (ST) are similarly defined (see [Ki1] for definition). The following properties of viscosity solutions are frequently used in our paper.

Theorem 2.7. (comparison principle, [Ki2]) Let $u, v$ be respectively viscosity sub- and supersolutions (of $(H S)$ or $(S T)$ ) in $D \times(0, T) \subset Q$ with initial data $u_{0} \prec v_{0}$ in $D$. If $u \leq v$ on $\partial D$ and $u<v$ on $\partial D \cap \bar{\Omega}(u)$ for $0 \leq t<T$, then $u(\cdot, t) \prec v(\cdot, t)$ in $D$ for $t \in[0, T)$.

Theorem 2.8. (a) For a given domain $\Omega_{0}$ in $\mathbb{R}^{n}$, there are the maximal and minimal viscosity solutions of (HS) in $Q$ with boundary data 1 and initial data $u_{0}$. If the minimal viscosity solution does not have an initial waiting time, then it is the unique viscosity solution of (HS) with given boundary data.

(b) $u$ is harmonic in $\Omega(u)$. Indeed $u(x, t)=h_{t}(x)$, where

$$
h_{t}(x)=\inf \left\{v \in \mathcal{P} \text { with } v=1 \text { on } \partial K \text { and } v \geq 0 \text { on } \Gamma_{t}\right\} \text {. }
$$

where $\mathcal{P}$ is the set of superharmonic functions in $\Omega_{t}$ which are lower semicontinuous in $\bar{\Omega}_{t}$.

(c) For a given initial data $v_{0} \geq 0$ with its positive set $\Omega_{0}(v)$ bounded in $\mathbb{R}^{n}$ there are the maximal and minimal viscosity solutions of (ST) in $\mathbb{R}^{n} \times[0, \infty)$. If the minimal viscosity solution does not have an initial waiting time, then it is the unique viscosity solution of (ST) with given boundary data. 


\section{Remark}

It is an open question that whether or not there is a unique viscosity solution of (HS) or (ST) when there is a waiting time.

\section{Proofs of the main result: Hele-Shaw problem}

In this section we prove the main results for Hele-Shaw problem. Let $0 \in \Gamma_{0}$ and $K \subset B_{1 / 2}\left(-e_{n}\right) \subset \Omega \subset B_{10}(0)$. Throughout the paper we denote $A_{k}=B_{2^{-k}}(0)-B_{2^{-k-1}}(0)(k \geq 1)$.

The key observation that will be used throughout this section is that there is a waiting time at $0 \in \Gamma_{0}$ if and only if there exists $t_{0}>0$ such that $\Gamma_{t_{0}} \cap A_{k} \neq \varnothing$ for every $k \geq 1$. In order to decide whether there exists such $t_{0}>0$ or not, we will use induction and estimate the change of the harmonic measure for each annulus $A_{k}$ in time. First we prove Theorem 1.3.

Definition 3.1. $\omega(x, \cdot, \Omega)$ is the unique probability measure on $\partial \Omega$ such that $\omega(x, E, \Omega)=w(x)$ where $w$ is the harmonic function in $\Omega$, which has boundary value 1 on $E$ and 0 elsewhere on its boundary.

First we state properties of NTA domains we use in the proof.

Lemma 3.2. ([JK], Lemma 4.1) Let $\Omega$ be NTA contained in $B_{10}(0)$. There exists a dimensional constant $\beta_{n}>0$ such that for any $\zeta \in \partial \Omega, 0<2 r<1$ and positive harmonic function $u$ in $\Omega \cap B_{2 r}(\zeta)$, if $u$ vanishes continuously on $B_{2 r}(\zeta) \cap \partial \Omega$, then for $x \in \Omega \cap B_{r}(\zeta)$,

$$
u(x) \leq C\left(\frac{|x-\zeta|}{r}\right)^{\beta_{n}} \sup \left\{u(y): y \in \partial B_{2 r}(\zeta) \cap \Omega\right\}
$$

where $C$ depends only on the NTA constant.

Lemma 3.3. ([JK], Lemma 4.11) Let $\Omega$ be NTA contained in $B_{10}(0)$. For $\zeta_{0}, \zeta \in \partial \Omega$ and $r<1$, let $B_{s}(\zeta) \subset B_{r / 2}\left(\zeta_{0}\right)$. If

$x \in \Omega-B_{2 r}\left(\zeta_{0}\right)$ and $x_{0}$ is a point in the middle of $B_{r}\left(\zeta_{0}\right) \cap \Omega$, then

$$
\omega\left(x_{0}, B_{s}(\zeta), \Omega\right) \approx \frac{\omega\left(x, B_{s}(\zeta), \Omega\right)}{\omega\left(x, B_{r}\left(\zeta_{0}\right), \Omega\right)}
$$

where $C_{1} \approx C_{2}$ means that the ratio between $C_{1}$ and $C_{2}$ is bounded above and below by a constant that depends on the NTA constant.

Proof of Theorem 1.3.

Recall that $I_{i}(i \geq 1)$ is the component of $\Omega_{0} \cap \partial B_{2^{-i}}(0)$ which separates 0 


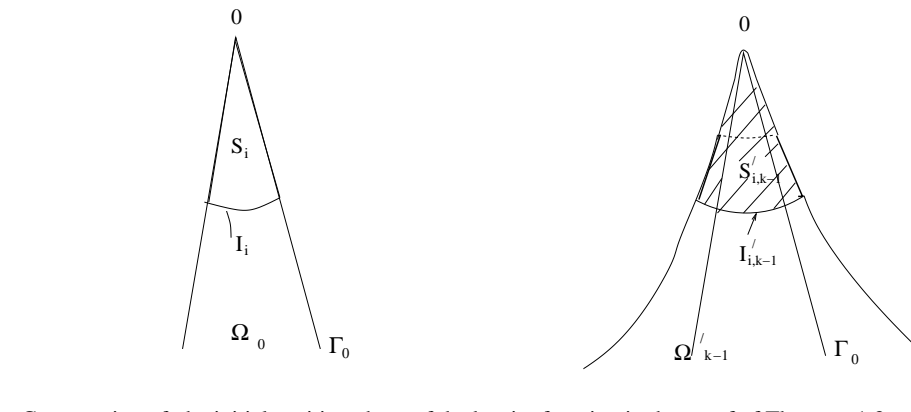

Figure 4 .

from $-e_{n}$. Denote by $M$ the NTA constant of $\Omega$. Let $S_{i}$ be the component of $\Omega_{0}-I_{i}$, which contains 0 on its boundary (see Figure 4 ).

Let $a=c_{0} \epsilon^{1 / \beta_{n}}$ where $c_{0}=c_{0}(M)$ will be determined later and $\beta_{n}$ is the dimensional constant given in Lemma 3.2. We will show that there exists $t_{0}>0$ such that for every $i \geq 1$,

$$
\Gamma_{t_{0}} \subset\left(\cup_{j=1}^{i} \mathcal{N}_{j}\right) \cup \tilde{\mathcal{N}}_{i+1}
$$

where $\mathcal{N}_{j}$ is the $a 2^{-j}$-neighborhood of $\Gamma_{0} \cap \partial\left(S_{j}-S_{j+1}\right)$ and $\tilde{\mathcal{N}}_{i+1}$ is the $a 2^{-i}$-neighborhood of $\Gamma_{0} \cap \partial\left(S_{i+1}\right)$.

Let us choose $t_{0}$ such that

$$
t_{0}=c_{1} a
$$

where $c_{1}=c\left(M, c_{0}\right)$ will be also determined later.

By a barrier argument, we can see that (3.1) holds when $i=1$ for $t_{0}$ if $c_{1}$ is chosen small enough. We will use an induction for the proof of (3.1). Assume that (3.1) holds for $i=k-1$. We will show that (3.1) also holds with the same $t_{0}$ for $i=k$.

Since $\Omega_{0}$ is an NTA domain, if we let $a \ll c(M)$ and if (3.1) holds for $i=k-1$, then we can see that $\Omega_{t_{0}}$ is contained in a NTA domain $\Omega_{k-1}^{\prime}$ such that (3.1) is satisfied with $\Gamma_{t_{0}}$ replaced by $\partial \Omega_{k-1}^{\prime}$ and for $i=k-1$.

Let $v$ be a harmonic function in $\Omega_{k-1}^{\prime}-K$ with boundary value 1 on $\partial K$ and 0 on $\partial \Omega_{k-1}^{\prime}$, then $u\left(x, t_{0}\right) \leq v(x)$. We will show that $v\left(x_{k}\right) \leq\left|x_{k}\right|^{2+\epsilon^{\prime}}$ for some $\epsilon^{\prime}>0$. (Recall that $x_{k}$ is a point on $I_{k}$ such that $B_{2^{-i} / M^{2}}\left(x_{k}\right) \subset \Omega_{0}$.)

Define $I_{i, k-1}^{\prime}$ be the component of $\Omega_{k-1}^{\prime} \cap \partial B_{2^{-i}}(0)$, which contains $I_{i}$ and let $S_{i, k-1}^{\prime}$ be the component of $\Omega_{k-1}^{\prime}-I_{i, k-1}^{\prime}$, which contains $S_{i}$ (see Figure 4.) For simplicity we will omit the $k$ - dependence on $I_{i, k-1}^{\prime}$ and $S_{i, k-1}^{\prime}$ from 
now on. Let

$$
\Omega_{i}=\Omega_{0} \cup S_{i}^{\prime}
$$

and let $v_{i}$ be the harmonic function in $\Omega_{i}$ with boundary value 1 on $\partial K$ and 0 on $\partial \Omega_{i}-\partial K$. Observe that $v_{i-1}-v_{i}$ is a positive harmonic function in $\Omega_{i}$ with boundary value $v_{i-1}$ on $\partial \Omega_{i} \cap \Omega_{i-1}$ and 0 elsewhere on its boundary.

Claim 1. Assume that (3.1) holds for $i=k-1$. Then for $2 \leq i \leq k$

$$
\frac{v_{i-1}\left(x_{k}\right)}{v_{i}\left(x_{k}\right)} \leq 1+C a^{\beta_{n}}
$$

where $C$ depends only on the NTA constant $M$.

Proof of Claim 1. Let $2 \leq i \leq k$. Since $S_{i-1}^{\prime}-S_{i}^{\prime}$ is contained in the $2 a 2^{-(i-1)}$-neighborhood of $\partial \Omega_{i-1}$, Lemma 3.2 implies that for $y \in \partial \Omega_{i} \cap$ $\Omega_{i-1}$

$$
\left\{\begin{aligned}
v_{i-1}(y) & \leq C\left(\frac{\operatorname{dist}\left(y, \partial \Omega_{i-1}\right)}{2^{-(i-1)}}\right)^{\beta_{n}} \sup _{x \in S_{i-1}^{\prime}-S_{i}^{\prime}} v_{i-1}(x) \\
& \leq C a^{\beta_{n}} \sup _{x \in S_{i-1}^{\prime}-S_{i}^{\prime}} v_{i-1}(x)
\end{aligned}\right.
$$

and

$$
\sup \left\{v_{i-1}(x): S_{i-1}^{\prime}-S_{i}^{\prime}\right\} \leq C v_{i-1}\left(x_{i-1}\right) .
$$

Also we obtain

$$
v_{i-1}\left(x_{i-1}\right) \approx v_{i}\left(x_{i-1}\right) .
$$

since Lemma 3.2 and Lemma 3.3 imply that for $1<j<i-1$ and $y \in \partial B_{2^{-j}}(0) \cap \Omega$

$$
\frac{\omega\left(y, \partial B_{2^{-j+1}}(0), \Omega^{i-1}\right)}{\omega\left(y, \partial B_{2^{-j+1}}(0), \Omega^{i}\right)} \leq 1+C 2^{(j-i) \beta_{n}} .
$$

Since $v_{i-1}-v_{i}$ is a positive harmonic function in $\Omega_{i}$ with boundary value $v_{i-1}$ on $\partial \Omega_{i} \cap \Omega_{i-1}$ and 0 elsewhere on its boundary, (3.2), (3.3) and (3.4) imply that

$$
\begin{aligned}
v_{i-1}\left(x_{k}\right)-v_{i}\left(x_{k}\right) & \leq \omega\left(x_{k}, \partial B_{2^{-i}}(0), B_{2^{-i}}(0) \cap \Omega_{i}\right) \sup _{\partial \Omega_{i} \cap \Omega_{i-1}} v_{i-1}(y) \\
& \leq C a^{\beta_{n}} \omega\left(x_{k}, \partial B_{2^{-i}}(0), B_{2^{-i}}(0) \cap \Omega_{i}\right) v_{i-1}\left(x_{i-1}\right) \\
& \approx C a^{\beta_{n}} \omega\left(x_{k}, \partial B_{2^{-i}}(0), B_{2^{-i}}(0) \cap \Omega_{i}\right) v_{i}\left(x_{i-1}\right) \\
& \leq C a^{\beta_{n}} v_{i}\left(x_{k}\right) .
\end{aligned}
$$


for some $C>0$ depending on the NTA constant $M$.

Claim 1 combined with $\frac{v\left(x_{k}\right)}{v_{1}\left(x_{k}\right)} \leq C$ and $\frac{v_{k}\left(x_{k}\right)}{u_{0}\left(x_{k}\right)} \leq C$ yields that

$$
\frac{v\left(x_{k}\right)}{u_{0}\left(x_{k}\right)} \leq C_{1}\left(1+C_{2} a^{\beta_{n}}\right)^{k}
$$

where $C_{1}$ and $C_{2}$ depend only on $M$.

Hence if we choose $c_{0}$ in the definition of $a$ small enough such that $C_{2} a^{\beta_{n}}<(1 / 10) \epsilon$, then

$$
v\left(x_{k}\right) \leq C u_{0}\left(x_{k}\right)(1+(1 / 10) \epsilon)^{k} \leq C\left|x_{k}\right|^{2+\epsilon}(1+(1 / 10) \epsilon)^{k} \leq C\left|x_{k}\right|^{2+\epsilon^{\prime}}
$$

for $\epsilon^{\prime}=\epsilon-\log _{2}(1+(1 / 10) \epsilon)>0$.

Now using (3.5) and the relation $u\left(x, t_{0}\right) \leq v(x)$, we prove $(3.1)$ for $i=k$. Let $B$ be a ball in $\Omega_{0}^{c}$ such that

$$
(1 / M)(a / 2) 2^{-k} \leq \operatorname{rad}(B) \leq \operatorname{dist}\left(B, S_{k}\right) \leq(a / 2) 2^{-k} .
$$

Let $(c(M) / a) B$ be the concentric ball containing $B$ with the radius of $(c(M) / a) \operatorname{rad}(B)$. Let $\phi$ be a radially symmetric solution of (HS) in $\Sigma:=$ $(c(M) / a) B \times\left[0, t_{0}\right)$ with fixed boundary data $C\left(2^{-k}\right)^{2+\epsilon^{\prime}}$ on $\partial((c(M) / a) B)$ and with initial positive set $(c(M) / a) B-B$. Then (3.5) and Lemma 3.2 imply that $u \leq \phi$ on the parabolic boundary of $\Sigma$. Hence by Theorem 2.7 $u \leq \phi$ in $\Sigma$. Since

$$
t_{0}:=c_{1} a \leq c_{1} a 2^{\epsilon^{\prime} k},
$$

it follows from the comparison with $u$ and $\phi$ that if we choose $c_{1}$ small enough then (3.1) holds for $i=k$. Now we can conclude.

\section{Proof of Theorem 1.4}

We will prove that for both (a) and (b), for any $t>0$ there is $k_{0}=k_{0}(t)$ such that $\Gamma_{t}(u) \cap B_{k_{0}}(0)=\varnothing$. First let us assume (a). Due to the Corkscrew condition on $\Omega$, for sufficiently big $n>0$, there exists $y_{n}$ such that $\left|y_{n}\right| \simeq$ $\left|x_{n}\right|$ and $B_{\left|x_{n}\right| / M}\left(y_{n}\right) \subset \Omega_{0}$. Moreover due to Lemma 3.2 and the Harnack inequality,

$$
u_{0}(x) \geq C\left|x_{n}\right|^{2-\epsilon_{n}} \text { in } B_{\left|x_{n}\right| / 2 M}\left(y_{n}\right) .
$$

Now if one considers a radially symmetric solution $\phi$ of (HS) with $K=$ $B_{\left|x_{n}\right| / 2 M}\left(y_{n}\right)$ and $\Omega=B_{\left|x_{n}\right| / M}\left(y_{n}\right), \phi \leq u$ by Theorem 2.7. Since $|D \phi| \geq$ $C\left|x_{n}\right|^{1-\epsilon}$ on $\Gamma_{t}(\phi)$ up to $t=t_{0}$ when $\Gamma_{t_{0}}(\phi)=B_{2\left|x_{n}\right|}\left(y_{n}\right)$, one obtains 


$$
t_{0} \leq C \frac{2\left|x_{n}\right|}{\left|x_{n}\right|^{1-\epsilon}}=C\left|x_{n}\right|^{\epsilon} .
$$

Due to Theorem 2.7, $\Omega_{t}(\phi) \subset \Omega_{t}(u)$ for each $t>0$ and it follows that $\Gamma_{t}(u) \cap B_{\left|x_{n}\right|}(0)=\varnothing$ if $t>t_{0}$. Since $t_{0} \rightarrow 0$ as $n \rightarrow \infty$ we can conclude.

Now for the case (b), for any sufficiently small $r>0$ due to Definition 1.1 condition 2 , there is $1 / M<m<2$ and $y \in \Omega$ such that $B_{m r}(y) \subset \Omega$ and $\bar{B}_{m r}(y) \cap \partial \Omega$ is nonempty. Let $\phi$ be a radially symmetric solution of (HS) with $\Omega=B_{m r}(y), K=B_{m r / 2}(y)$ and $f=r^{2}$. By comparing $u$ to $\phi$ for each $r>0$, it follows that for any $t_{0}>0$ there is $k_{0}>0$ such that for $k>k_{0}$ a fixed portion of $\Gamma_{0}(u)$ in $A_{k}$ expands in the direction of $e_{n}=(0, . ., 1) \in \mathbb{R}^{n}$ by distance $C 2^{-k}$. In particular if $0 \in \Gamma_{t_{0}}(u)$, then for any $t_{0}>0$ there exists $\epsilon>0$ such that

$$
\sup _{x \in I_{i}} u\left(x, t_{0}\right) \geq\left|2^{-i}\right|^{2-\epsilon} \text { for sufficiently large } i .
$$

Hence by (a) $u\left(0, t_{0}+s\right)>0$ for any $s>0$. Since $t_{0}$ is arbitrary, we can conclude.

\section{Proof of Proposition 1.6.}

Let $t_{0}>0$ be sufficiently small. Suppose that there exist $a_{i}<\frac{m}{10}$ $(0 \leq i \leq k-1)$ such that $\Gamma_{t_{0}} \cap A_{i}$ is contained in the $a_{i} 2^{-i}$-neighborhood of $\Gamma_{0} \cap A_{i}$ for $0 \leq i \leq k-1$. By a similar argument as in Claim 1

$$
u\left(x_{k}, t_{0}\right) \leq C \Pi_{i=0}^{k-1}\left(1+a_{i}\right) u\left(x_{k}, 0\right)
$$

since we can take the dimensional constant $\beta_{n}$ in Claim 1 as 1 due to our extra assumption on $\Omega_{0}$.

A barrier argument as in the proof of Theorem 1.3 yields that $\Gamma_{t_{0}} \cap A_{k}$ is contained in the $a_{k} 2^{-k}$-neighborhood of $\Gamma_{0} \cap A_{k}$ where

$$
a_{k}:=C \frac{\Pi_{i=0}^{k-1}\left(1+a_{i}\right) u\left(x_{k}, 0\right)}{2^{-2 k}} \cdot t_{0} .
$$

Observe that if we can show $a_{k}<\frac{m}{10}$, then by induction there exists a waiting time at 0 . Since we can take $t_{0}>0$ sufficiently small, we can conclude that if $\lim \sup \tilde{a}_{k}<\infty$ for the sequence $\tilde{a}_{k}=\Pi_{i=0}^{k-1}\left(1+\tilde{a}_{i}\right) 2^{2 k} u\left(x_{k}, 0\right)$, $\tilde{a}_{0}=1$, then there exists a waiting time.

(a) of Theorem 1.5 follows from Proposition 1.6. Next, we prove the second part of Theorem 1.5. 
Definition 3.4. For $\zeta \in \Gamma_{0}$, let

$$
H(\zeta, t):=\operatorname{dist}\left(\zeta, \Gamma_{t}\right)
$$

\section{Proof of (b) of Theorem 1.5.}

1. Let $t_{0}>0$ and partition $\left[0, t_{0}\right]$ into $0=s_{0}<s_{1}<\cdots<s_{P}=t_{0}$. By a simple barrier argument with a radially symmetric test function we obtain that

$$
H\left(\zeta, s_{1}\right) \geq C \frac{u\left(x_{i}, 0\right)}{2^{-i}} \cdot s_{1}
$$

for every $\zeta \in \Gamma_{0} \cap A_{i}$. Hence by a similar argument as in Claim 1

$$
u\left(x_{k}, s_{1}\right) \geq \Pi_{i=0}^{k-1}\left(1+C \frac{u\left(x_{i}, 0\right)}{2^{-2 i}} \cdot s_{1}\right) \cdot u\left(x_{k}, 0\right) .
$$

2. Repeating our argument, we also obtain that for $1 \leq m \leq P$

$$
u\left(x_{k}, s_{m}\right) \geq \Pi_{i=0}^{k-1} \Pi_{j=0}^{m-1}\left(1+C \frac{u\left(x_{i}, s_{j}\right)\left(s_{j+1}-s_{j}\right)}{2^{-2 i}}\right) \cdot u\left(x_{k}, 0\right)
$$

and for any $\zeta \in \Gamma_{0} \cap A_{k}$

$$
H\left(\zeta, s_{P}\right) \geq C \sum_{m=0}^{n-1} \frac{u\left(x_{k}, s_{m}\right)\left(s_{m+1}-s_{m}\right)}{2^{-k}}
$$

3. In terms of integration, (3.6) yields

$$
\begin{aligned}
u\left(x_{k}, t\right) & \geq u_{0}\left(x_{k}\right) \Pi_{i=1}^{k-1}\left(1+C 2^{2 i} \int_{0}^{t} u\left(x_{i}, s\right) d s\right) \\
& \geq 2^{-2 k} k^{-\alpha} t \sum_{i=1}^{k-1} 2^{2 i} u_{0}\left(x_{i}\right) \\
& \geq 2^{-2 k} k^{-\alpha} t \sum_{i=1}^{k-1} i^{-\alpha} \\
& \geq 2^{-2 k} k^{1-2 \alpha} t
\end{aligned}
$$

where the second and third inequality holds due to the assumption on the decay rate of $u$. Observe that $2 \alpha-1<\alpha$ since $\alpha<1$. Hence if we define a sequence $b_{1}, b_{2}, \ldots$ such that $b_{1}=\alpha$ and $b_{n+1}=2 b_{n}-1$, then there exists $l=l(\alpha)$ such that $b_{l} \leq 0$. 
4. Now we are ready to show that there is no waiting time for $u$ at the origin. Let us fix $t_{0}>0$ and define

$$
b_{j}=j \frac{t_{0}}{2 l}, j=0, . ., l .
$$

Then by inductively applying the estimate (3.8) replacing $u\left(x_{k}, t\right)$ by $u\left(x_{k}, b_{j}\right)$ and $u\left(x_{i}, 0\right)$ by $u\left(x_{i}, b_{j-1}\right)$ for $j=1,2, \ldots, l$ we have

$$
u\left(x_{k}, \frac{t_{0}}{2}\right)=u\left(x_{k}, b_{l}\right) \geq 2^{-2 k}\left[\Pi_{i=1}^{l}\left(b_{i}-b_{i-1}\right)^{2^{l-i}}\right] k^{-b_{l}} \geq 2^{-2 k}\left(\frac{t_{0}}{2 l}\right)^{2^{l}}
$$

In other words $u$ decays slower than quadratically at $t=t_{0} / 2$. Hence due to Theorem $1.3, u\left(0, t_{0}\right)>0$. Since $t_{0}$ is arbitrary, we can conclude.

\section{Proofs of the main result: Stefan problem}

In this section we assume $\Gamma_{0}$ to be locally Lipschitz along the direction $e_{n}=(0, . ., 0,1)$ in a neighborhood of the vertex 0 . More precisely in a small neighborhood of the origin we assume that $\Omega_{0}$ is given as $\left\{\left(x^{\prime}, x_{n}\right): x_{n}>\right.$ $\left.f\left(x^{\prime}, 0\right)\right\}$ where $f$ is Lipschitz with Lipschitz constant $L$.

The following lemmas are important in our analysis in this section. In particular, Lemma 4.3 shows that the initial heat with fixed Lipschitz positive phase changes to be (almost) harmonic over the amount of time $t \sim d^{2}$, where $d$ is the distance between the given point in the positive phase and the free boundary. In our free boundary problem (ST), this change will affect the expansion of the initial positive phase over time and vice versa. Lemma 4.1 gives us a control over the change of the initial heat before it changes to be (almost) harmonic, that is for the time interval $0 \leq t \leq d^{2}$.

Lemma 4.1. [Di]

Suppose $u_{0}(x) \in C_{0}\left(\mathbb{R}^{n}\right) \cap C^{\delta}\left(\mathbb{R}^{n}\right)$ for some $\delta>0$ and let $u(x, t)$ solve the heat equation in $\mathbb{R}^{n} \times[0, \infty)$ with initial data $u_{0}$. Then there exists a constant $A$ depending only on $n, \delta$ and the Hölder constant of $u_{0}$ in $B_{1}(0)$ such that

$$
\left|u_{t}\right|(x, t)+\left|u_{x_{i} x_{j}}\right|(x, t) \leq A t^{\delta / 2-1}
$$

in $B_{1}(0) \times(0,1)$ for any $i, j=1,2, \ldots n$.

Let $Q_{a}$ be the $a$-cube $B_{a}(0) \times(-a, a)$ in $\mathbb{R}^{n+1}$. 
Lemma 4.2. [ACS] Let $u$ be a caloric function in $Q_{1} \cap D$, where $D \cap Q_{1}=\left\{x_{n}>f\left(x^{\prime}, t\right)\right\}$ where $f$ is Lipschitz and $(0,0) \in \partial D$. Then there exists a constant $\delta>0$ depending on $n$, the Lipschitz constant $L$ of $D$, and the ratio $r$ between the supremum of $u$ in $Q_{1}$ and $u\left(-e_{n}, 0\right)$ such that $\nabla u \cdot e_{n} \geq 0$ in $D \cap Q_{\delta}$.

Lemma 4.3. [ACS] Let $u, D$ and $r, L$ be as in Lemma 4.3. Then there exist $\epsilon, \delta>0$ depending on $n, r, L$ such that

$$
u+u^{1+\epsilon} ; \quad u-u^{1+\epsilon}
$$

are subharmonic and superharmonic, respectively, at each time in $Q_{\delta} \cap D$.

Lemma 4.4. (Dahlberg, see $[D]$ ) Let $u_{1}, u_{2}$ be two nonnegative harmonic functions in a domain $D$ of $\mathbb{R}^{n}$ of the form

$$
D=\left\{\left(x^{\prime}, x_{n}\right) \in \mathbb{R}^{n-1} \times \mathbb{R}:\left|x^{\prime}\right|<2,\left|x_{n}\right|<2 M, x_{n}>f\left(x^{\prime}\right)\right\}
$$

with $f$ a Lipschitz function with constant less than $M$ and $f(0)=0$. Assume further that $u_{1}=u_{2}=0$ along the graph of $f$. Then for

$$
D_{1 / 2}=\left\{\left|x^{\prime}\right|<1,\left|x_{n}\right|<M, x_{n}>f\left(x^{\prime}\right)\right\}
$$

We have

$$
0<C_{1} \leq \frac{u_{1}\left(x^{\prime}, x_{n}\right)}{u_{2}\left(x^{\prime}, x_{n}\right)} \cdot \frac{u_{2}(0, M)}{u_{1}(0, M)} \leq C_{2}
$$

with $C_{1}, C_{2}$ depending only on $M$.

Lemma 4.5. (Caffarelli, see [C1]) Let $u$ be as in Lemma 4.4. Then there exists $c>0$ depending only on $M$ such that for $0<d<c u_{n}(0, d):=$ $\frac{\partial u}{\partial x_{n}}(0, d) \geq 0$ and

$$
C_{1} \frac{u(0, d)}{d} \leq u_{n}(0, d) \leq C_{2} \frac{u(0, d)}{d}
$$

where $C_{i}=C_{i}(M)$.

Let $u$ and $h_{0}$ to be as in section 1.2.

Lemma 4.6. If $h_{0}(x)$ has less than quadratic decay at 0 , then there is no waiting time for $u$. 


\section{Proof}

1. First we choose a small $t_{0}>0$. Below we will show that, $u(0, t)>0$ for $t>t_{0}$. Since $t_{0}$ is arbitrary we can then conclude. Also without loss of generality, we may assume that $\Gamma_{0}$ is Lipschitz in $B_{1}$ along the direction $e_{n}$.

2 . Let $w$ solve the heat equation in the domain

$$
\Sigma:=\Omega_{0} \times\left[0, t_{0}\right]
$$

with initial data $u(x, 0)$. Since $\Sigma$ is Lipschitz in space and time, In a small neighborhood $B_{h}(0) \times\left[t_{0}, t_{0}+h^{2}\right], h=h\left(t_{0}\right)$ there is $\epsilon$ depending only on the Lipschtz constant of $\Gamma_{0}$ in $B_{1}$ such that $w_{1}:=w-w^{1+\epsilon}$ is superharmonic in $\Sigma$.

3. Let us choose $C=C\left(t_{0}\right)$ large enough that $C w_{1} \geq w$ at $\left(-h e_{n}, t_{0}\right)$. Then by continuity of $w$ in time and by Lemma 4.4 applied at each $t$,

$$
w \geq w_{1} \geq C h_{0} \text { in } B_{h}(0) \times\left[t_{0}, t_{0}+\epsilon\right]
$$

for small $\epsilon>0$.

In particular it follows that, for some $k>0$,

$$
w\left(-r e_{n}, t\right) \geq C h_{0}\left(-r e_{n}, t\right) \geq C h_{0}\left(-r e_{n}, 0\right)>C r^{2-k} \text { for } t \in\left[t_{0}, t_{0}+\epsilon\right] .
$$

4. Lastly, observe that $w \leq u$ by the maximal principle of the heat equation. Hence for sufficiently small $r>0$

$$
u\left(-r e_{n}, t\right) \geq C r^{2-k} \text { for } t \in\left[t_{0}, t_{0}+\epsilon\right] .
$$

Since $\Omega_{0}$ is Lipschitz, $B_{C r}\left(-r e_{n}\right) \subset \Omega_{0}$.

Next we consider a radially symmetric subsolution $\phi(x, t)$ of $(\mathrm{ST})$ in $\Sigma:=\left(\mathbb{R}^{n}-B_{C r / 2}\left(-r e_{n}\right)\right) \times\left[t_{0}, t_{0}+\epsilon\right]$ whose initial and fixed boundary data are given as

$$
\begin{aligned}
& -\Delta \phi\left(x, t_{0}\right)=0 \text { in } B_{C r}\left(-r e_{n}\right)-B_{C r / 2}\left(-r e_{n}\right), \\
& \phi\left(\cdot, t_{0}\right)=0 \text { on } \partial B_{C r}\left(-r e_{n}\right), \quad \phi(\cdot, t)=C r^{2-k} \text { on } \partial B_{C r / 2}\left(-r e_{n}\right) .
\end{aligned}
$$

By comparing $u$ and $\phi$ in $\Sigma$, it follows that

$$
u\left(0, t_{0}+r^{k / 2}\right)>0 \text { for sufficiently small } r>0 .
$$

Hence we conclude. 
Proving the occurrence of the waiting time is more involved since we need to observe the behavior of the solution during a time period $0<t<t_{0}$.

\section{Proof of Theorem 1.8}

1. Recall that we assume $u_{0}(x) \in C^{\delta}\left(\bar{\Omega}_{0}\right)$ and the minimal and maximal decay rate $a$ and $b$ of $h_{0}$ satisfies $2<\alpha_{1} \leq \alpha_{2}$ and $\alpha_{2}-\alpha_{1}<\delta / 2$, where $h_{0}$ is the harmonic function in $\Omega_{0}-K, K$ a compact subset of $\Omega$ with boundary value 0 on $\partial \Omega_{0}$ and 1 on $\partial K$. Throughout the proof we will denote $C$ as a positive constant which only depends on $\delta, L$ and $n$.

2. The plan is to construct a supersolution $\tilde{v}$ of (ST) in a neighborhood of the origin which has a waiting time at the origin and to compare $\tilde{v}$ with $u$ to conclude our theorem. For this purpose first we will construct a supersolution $\tilde{h}$ of (HS) such that $u \leq \tilde{v} \leq \tilde{h}$ in a small neighborhood of 0 and for a small time.

3. Let $r>0$ be sufficiently small. First we construct a supersolution $h(x, t)$ of $(\mathrm{HS})$ in $B_{r}(0) \times[0,1)$ such that

(i) $\Omega_{0}(u) \cap B_{r}(0) \subset \Omega_{0}(h) \cap B_{r}(0)$ and $\bar{\Omega}_{0}(u) \cap \partial B_{r}(0) \subset \Omega_{0}(h)$;

(ii) $\Gamma(h)$ has a waiting time at $(0,0)$;

(iii) $\beta_{2}-\beta_{1}<\delta / 2$, where $\beta_{1}$ and $\beta_{2}$ are the maximal and minimal rate of $h(x, 0)$;

(iv) $\Gamma(h)$ is Lipschitz in space-time.

Note that we cannot use a solution of (HS) with initial free boundary $\Gamma_{0}$ as a barrier for $u$ most importantly since we do not know if it satisfies property (iv) and hence we cannot apply Lemma 4.2 and 4.3.

4. To construct such $h$ satisfying (i)-(iv), first we choose a sufficiently small $a>0$ and define $\Omega^{\prime}$ to be the support of the following function:

$$
U(x):=\sup _{y \in B_{2 a|x|}(x)} u(y, 0) .
$$

Since $h_{0}(x)$ has its minimal decay rate more than 2 near the origin, the harmonic function in $\Omega^{\prime}-K$ with boundary value 0 on $\partial \Omega^{\prime}$ and 1 on $\partial K$ has a minimal decay rate $2+\epsilon^{\prime}$ for some $\epsilon^{\prime}>0$, if $a>0$ is sufficiently small. (Its proof is the same as that of Theorem 1.2.) Next for $0 \leq t \leq 1$ we define 


$$
U^{t}(x):=\inf _{y \in B_{a(t)|x|}(x)} U(y)
$$

where $a(t):=2 a-\frac{a}{2} t|x|^{\epsilon^{\prime} / 2}$. Next we let

$$
\Omega_{t}^{\prime}=\Omega\left(U^{t}\right)
$$

and let $h^{t}(x)$ be the harmonic function in $\Omega_{t}^{\prime}-K$ with boundary data 0 on $\partial \Omega_{t}^{\prime}$ and 1 on $K$.

If we define $h(x, t):=h^{t}(x)$, then (i),(ii),(iv) follow from its construction and (iii) is also satisfied if $a$ is sufficiently small, since $2+\epsilon^{\prime} \rightarrow \alpha_{1}$ as $a \rightarrow 0$ due to the proof of Theorem 1.2.

Now we show that $h(x, t)$ is a supersolution of $(\mathrm{HS})$ in $B_{r}(0) \times[0,1]$. Note that $h(x, t) \leq|x|^{2+\epsilon^{\prime}}$. Hence due to the exterior ball property of $\Omega_{t}^{\prime} \cap B_{r}(0)$ and by Lemma 4.5 ,

$$
\left|\nabla_{x} h\right| \leq C|x|^{1+\epsilon^{\prime}} \text { on } \Gamma_{t}(h) \cap B_{r}(0)
$$

where $C$ depends on $L, n$ and $a$.

On the other hand the normal velocity of $\Gamma_{t}(h)$ is given as $a|x|^{1+\epsilon^{\prime} / 2}$ by its construction. Therefore for sufficiently small $r>0$ it follows that $h$ is a supersolution of $(\mathrm{HS})$ in $B_{r}(0) \times[0,1]$ with property (i)-(iv).

5 . Recall that $\beta_{1} \leq \beta_{2}$ is given as respectively the minimal and maximal decay rate of $h(x, 0)$ near the origin and $\beta_{2}-\beta_{1}<\delta / 2$ by our construction of $h$. We. will first prove the theorem for the case $2<\alpha_{1} \leq \alpha_{2}<2+\delta$. Note that in this case we have $\beta_{2}<\alpha_{2}<2+\delta$.

Let us fix $0<l<<r$ and let $\alpha(t)$ be given as

$$
\alpha(t)=t^{\delta / 2-\beta_{2} / 2}
$$

on $\left[0, t_{0}\right]$, where $t_{0}$ is chosen such that $\int_{0}^{t_{0}} \alpha(t) d t=1$. Note that $\alpha(t)$ is integrable since $\delta-\beta_{2}>-2$ by our hypothesis.

With above choice of $\alpha(t)$

$$
\tilde{h}(x, t):=\alpha(t) h\left(x-l e_{n}, \int_{0}^{t} \alpha(t) d t\right) .
$$

is also a supersolution of (HS) in $B_{r}(0) \times\left[0, t_{0}\right]$. Observe that $\tilde{h}$ has a waiting time at the vertex $l e_{n}$. 
6. Now we construct a supersolution $\tilde{v}$ of $(\mathrm{ST})$ such that $u \leq \tilde{v}$ for a small time and $\tilde{v}$ has a waiting time. Let $v$ solve the heat equation in $\Omega(\tilde{h})$ with a proper initial data $v_{0}(x)$ to be chosen. We would like to construct the initial data $v_{0}(x)$ whose support lies inside $\Omega_{0}(\tilde{h})$ such that $v_{0}$ and $v$ satisfies

(i) $v(x, 0) \in C^{\delta}\left(\mathbb{R}^{n}\right)$,

(ii) $u \prec v$ on the parabolic boundary of the following set:

$$
\mathcal{O}:=\left\{(x, t):\left|x-l e_{n}\right| \leq t^{1 / 2}, 0 \leq t \leq t_{0}\right\} .
$$

Without loss of generality we may assume that

$$
u_{0}(x) \leq c_{0}|x|^{\delta}
$$

where $c_{0}=c_{0}(\delta, L, n)$ is sufficiently small constant which will be chosen later. Since $u_{0} \in C^{\delta}\left(\mathbb{R}^{n}\right)$ and $u_{0}(0)=0$. For simplicity we may assume $C=1$. Let $\phi(x)=\phi(|x|)$ to be a smooth function such that $0 \leq \phi \leq 1$, $\phi=1$ on $|x| \leq 1 / 2$ and $\phi=0$ if $|x| \geq 1$. We then let

$$
v_{0}(x)=\int_{\Omega_{0}}|y|^{\delta} \phi\left(\frac{x-y}{g(x)}\right) d y
$$

where $g(x)=a|x|+l$. Observe that, since $l<<r$, the support of $v_{0}$ is contained in $\Omega(\tilde{h}) \cap \mathcal{O}$.

Note that $u_{0} \prec c_{0} v_{0}$ by properties of $\phi$ and $g$. Moreover from a straightforward computation it follows that $v_{0}(x) \in C^{\delta}\left(\mathbb{R}^{n}\right)$.

7. First we show that $u \prec c_{1} v$ on the lateral boundary of $\mathcal{O}$, where $c_{1}=C c_{0}$. Let $(y, s)$ be on the lateral boundary of $\mathcal{O}$, i.e., $s=a^{2}\left|y-l e_{n}\right|^{2}$. By (4.3) and Theorem 4.1

$$
u(y, s) \leq C c_{0}|y|^{\delta} .
$$

On the other hand due to Theorem 4.1,

$$
v(y, \tau) \geq C|y|^{\delta} \text { if } 0 \leq \tau \leq|y|^{2} \text { and if } d\left(y, \Omega_{0}\right) \leq(a / 2)|y| .
$$

We claim that for any $m>0$ and $0 \leq s \leq a^{2} m^{2}$,

$$
d\left(p, \Omega_{0}\right) \leq a m / 2 \text { if } p \in \Gamma_{s}(u) \cap \partial B_{m}(0) .
$$


If (4.4) holds, then from above estimates it would follow that $u(y, s)<$ $C c_{0} v(y, s)$ if $(y, s) \in \Omega(u)$.

To show (4.4), first note that by our hypothesis for any $m>0$ and $p \in \Gamma_{0}(u) \cap \partial B_{m}(0), B_{C_{1} a m}\left(p+a m e_{n}\right) \subset \Omega_{0}^{C}$ for $C_{1}$ depending on the Lipschitz constant of $\Gamma_{0}$. For $m>0$ let

$$
t(m)=\sup \left\{t>0: u\left(p+a m / 2 e_{n}, t\right)=0 .\right\}
$$

By a barrier argument we will show that

$$
t(m) \geq a^{2} m^{2}
$$

which proves (4.4).

Note that due to Theorem $4.1 u(y, \tau) \leq C c_{0}|y|^{\delta}$ for $0 \leq \tau \leq|y|^{2}$, and in particular

$$
u(\cdot, \tau) \leq C c_{0} m^{\delta} \text { on } B_{3 m}\left(p+a m e_{n}\right) \text { for } 0 \leq \tau \leq m^{2}
$$

Let $\phi$ be a supersolution of $(\mathrm{ST})$ in $\Sigma:=\left(\mathbb{R}^{n}-B_{C_{1} a m}\left(p+a m e_{n}\right)\right) \times$ $\left(0, m^{2}\right)$ whose initial and fixed boundary data are given as

$$
\begin{cases}-\Delta \phi(x, 0)=0 & \text { in } B_{3 m}\left(p+a m e_{n}\right)-B_{C_{1} a m}\left(p+a r e_{n}\right), \\ \phi(\cdot, 0)=0 & \text { on } \partial B_{C_{1} a m / 2}\left(p+a m e_{n}\right) \\ \phi(\cdot, t)=C c_{0} m^{\delta} & \text { on } \partial B_{3 m}\left(p+a m e_{n}\right) .\end{cases}
$$

Due to (4.6) $u$ is then less than $\phi$ on the lateral boundary of $\Sigma$. Moreover since $u_{0} \in C^{\delta}\left(\mathbb{R}^{n}\right), u_{0} \leq C_{3} \phi$ for sufficiently large $C_{3}$. Hence we can compare $u$ and $C_{3} \phi$ in $\Sigma$ and it follows that

$$
t(m) \geq \min \left[m^{2}, C_{4} a m^{2-\delta}\right] \geq a^{2} m^{2} .
$$

for sufficiently small $m$.

8. Next we show that for some $0<c_{2}<1, c_{2}$ only depends on Landn, $\tilde{v}(x, t):=c_{2} v(x, t)$ is a supersolution of (ST) in $\mathcal{O}$ for a proper choice of $\alpha(t)$. Since $\Gamma(v)=\Gamma(\tilde{h})$ and $\tilde{h}$ is a supersolution of $(\mathrm{ST})$ in $\mathcal{O}$, we only need to show that

$$
|D v(x, t)| \leq C|D \tilde{h}(x, t)| \text { on } \Gamma(v) .
$$

Observe that due to Theorem 4.1

$$
v\left((-s+l) e_{n}, t\right) \leq C s^{\delta}
$$


for $0 \leq t \leq s^{2}$. Thus we can apply Lemma 4.3 and obtain $\epsilon>0$ depending only on $L, a$ and $n$ such that $v_{1}=v+v^{1+\epsilon}$ is subharmonic in $B_{s}\left(l e_{n}\right)$ for any $z \in \Gamma_{a^{2} s^{2}}(v)$. Moreover by definition of $v_{1}$ we have

$$
v_{1}\left(x, a^{2} s^{2}\right) \leq C s^{\delta} \text { if } d\left(x, \Gamma_{a^{2} s^{2}}(v)\right) \leq a s .
$$

On the other hand, since $h$ increases in time,

$$
h\left(-s e_{n}, t\right) \geq h\left(-s e_{n}, 0\right) \geq s^{\beta_{2}}
$$

where $\beta_{2}$ is the maximal decay rate of $h(x, 0)$. By definition of $\alpha(t)$ and by previous estimates, for a given constant $C_{2}>0$ we can choose $C_{1}>0$ in the definition of $\alpha(t)$ such that we have

$$
C_{2} \tilde{h}(x, t) \geq v_{1}(x, t) \text { at }(x, t)=\left(-s e_{n}, a^{2} s^{2}\right), 0<s<t_{0} .
$$

Now due to Lemma 4.4, we have $C \tilde{h} \geq v_{1}$ in $\mathcal{O}$, and therefore $C \tilde{h} \geq v$ in $\mathcal{O}$. Since $C \tilde{h} \geq v$ and $\Gamma(v)=\Gamma(\tilde{h})$, we obtain $C|D \tilde{h}| \geq|D v|$ on $\Gamma(v)$. Hence it follows that there exists a positive constant $c_{2}=c_{2}(\delta, L, n)$ such that $\tilde{v}:=c_{2} v$ is a supersolution of $(\mathrm{ST})$ in $\mathcal{O}$.

9. Now we choose $c_{0}$ in (4.3) small enough such that $c_{1} \leq c_{2}$. Then by previous arguments it follows that $u \leq \tilde{v}$ on the parabolic boundary of $\mathcal{O}$. Now Theorem 2.7 yields that $u \leq \tilde{v}$ in $\mathcal{O}$, yielding that $u\left(l e_{n}, s\right)=0$ for $0 \leq s \leq t_{1}$, where $t_{1}$ is independent of $l$. Lastly we use the fact that $l$ is arbitrarily small to conclude that $u$ has a waiting time at 0 .

10. It remains to prove the theorem when $2+\delta \leq \alpha_{2}$. We claim that in this case one can always have a bigger Lipschitz domain $\Sigma$ which contains $\Omega$ with $0 \in \Sigma$ and the maximal and minimal decay rate $\alpha_{2}^{\prime}$ and $\alpha_{1}^{\prime}$ of the corresponding harmonic function satisfies that

$$
2<\alpha_{1}^{\prime} \leq \alpha_{2}^{\prime}<2+\delta .
$$

Then we can proceed as in previous step to conclude. Hence our last step is to prove our claim.

\section{Proof of the claim:}

Suppose that $2+\delta \leq \alpha_{2}$. Since $\alpha_{2}-\alpha_{1}<\delta / 2$ by our hypothesis, it follows that $\alpha_{1}, \alpha_{2}>2+\frac{\delta}{2}$. Let $h$ be a constant such that $0<h<\frac{\delta}{2}$ and

$$
\alpha_{2}=\alpha_{1}+\frac{\delta}{2}-h ; r^{\alpha_{2}} \leq \sup _{|x|=r} u(x) \leq r^{\alpha_{1}} .
$$


Let $\delta / 2<\delta^{\prime}<\delta$ and let

$$
\Omega_{1}=\Omega_{0} \cup\left(W\left(\frac{\pi}{2\left(2+\delta^{\prime}\right)},-e_{n}\right) \cap B_{1}(0)\right)
$$

where

$$
W(\theta, \nu)=\left\{x \in \mathbb{R}^{n}:<x, \nu>\geq|x| \cos (\theta / 2)\right\} .
$$

We also let $v_{1}(x)$ be the harmonic function in $\Omega_{1}-K$ with boundary data 1 on $\partial K$ and 0 on $\partial \Omega_{1}$. Then

$$
\sup _{|x|=r} v_{1}(x) \geq r^{2+\delta^{\prime}} \text { for } 0<r<1 \text { and } \delta / 2<\delta^{\prime}<\delta .
$$

If $\sup _{|x|=r} v_{1}(x) \leq r^{2+k}$ for some $k>0$, then let $\Sigma=\Omega_{1}$. If not then we inductively construct $\Sigma$ as follows. Let $z_{i}$ be a point in the middle of $A_{i} \cap W\left(\frac{\pi}{2\left(2+\delta^{\prime}\right)},-e_{n}\right)$ where $A_{i}=B_{2^{-i}}(0)-B_{2^{-i-1}}(0)$. Let $i_{1}>0$ be the smallest number such that $\left|z_{i_{1}}\right|^{2+\frac{\delta}{2}} \leq v\left(z_{i_{1}}\right) \leq 10\left|z_{i_{1}}\right|^{2+\frac{\delta}{2}}$. Let

$$
\Omega_{2}=\left(\Omega_{1}-B_{2^{-i_{1}}}(0)\right) \cup \Omega_{0}
$$

and let $v_{2}$ be the corresponding harmonic function in $\Omega_{2}-K$ with boundary value 1 on $\partial K$ and 0 on $\partial \Omega_{2}$. Then for $2^{-i_{1}}<r<1$

$$
c r^{2+\delta^{\prime}} \leq \sup _{|x|=r} v_{1}(x) \leq C r^{2+(\delta / 2)} .
$$

Since $\Omega_{0}$ is a Lipschitz domain, for

$$
x \in \Omega_{2} \cap B_{2^{-i_{1}}}(0)=\Omega_{0} \cap B_{2^{-i_{1}}}(0)
$$

we have

$$
\begin{aligned}
v_{2}(x) \approx & u(x) \cdot \frac{v_{2}\left(z_{i_{1}}\right)}{u\left(z_{i_{1}}\right)} \approx u(x) \cdot \frac{v_{2}\left(z_{i_{1}}\right)}{u\left(z_{i_{1}}\right)} \\
& \leq C|x|^{\alpha_{1}}\left|z_{i_{1}}\right|^{2+\frac{\delta}{2}-\alpha_{2}}=C|x|^{\alpha_{1}}\left|z_{i_{1}}\right|^{2-\alpha_{1}+h} \leq C|x|^{2+h} .
\end{aligned}
$$

If $v_{2}(x) \geq|x|^{2+\delta^{\prime}}$, then let $\Sigma=\Omega_{2}$. If not, we repeat above argument, i.e. let $i_{2}$ be the smallest number such that $i_{2}>i_{1}$ and $v_{2}\left(z_{i_{2}}\right) \approx\left|z_{i_{2}}\right|^{2+\delta / 2}$ and let

$$
\Omega_{3}=\Omega_{2} \cup\left(W\left(\frac{\pi}{2\left(2+\delta^{\prime}\right)},-e_{n}\right) \cap B_{2^{-i_{2}}}(0)\right) .
$$

Let $\Sigma$ be a region obtained inductively as above. At each steps $\Omega_{i}$ may not be Lipschitz, but with a slight modification of $\Omega_{i}$ we can get a Lipschitz domain without changing the minimal and maximal decay rates. 


\section{A counterexample for Theorem 1.8}

As in section 4 , let $u_{0} \geq 0, \Omega_{0}(u)=\left\{u_{0}>0\right\}$ be a Lipschitz domain and let $u$ be the viscosity solution of (ST) with initial data $u_{0}$. The proofs of Theorems 1.7 and 1.8 suggests that the initial positive phase $\Omega_{0}$ changes into a new phase while the initial data transforms into (almost) harmonic function, and the waiting time phenomena occurs when this new phase, associated with the corresponding harmonic initial data, has a waiting time at the vertex. Similar observation has been also made in the example of $[\mathrm{K}]$ mentioned in the introduction.

Based on above observation we will construct a counterexample for Theorem 1.8 where the harmonic function $h_{0}$ associated with $\Omega_{0}$ has more than quadratic decay at the origin and $u_{0} \in C^{\delta}\left(\mathbb{R}^{n}\right)$ for some $\delta>0$ but $u$ has no waiting time. The key idea is to construct $\Omega_{0}$ such that the change of the geometry of the positive phase caused by the initial heat $u_{0}$ is big enough to change the decay rate of the harmonic initial data in a drastic way.

Let $a$ be sufficiently small, let $A$ be sufficiently large. Define a sequence

$$
1>t_{1}>s_{1}>t_{2}>s_{2} \ldots
$$

by $s_{m}=a t_{m}^{1+\delta}$ and $t_{m+1}=s_{m} \cdot\left(\frac{s_{m}}{t_{m}}\right)^{A}$.

Let $E_{r}(x)=x+B_{r}(0) \cap\left\{y \in \mathbb{R}^{n}: y_{n}:=<y, e_{n}><0\right\}$ be a half-ball with center $x$ and radius $r$ and let

$$
\Omega_{0}=\left(B_{1}(0) \cap W\left(\frac{\pi}{10},-e_{n}\right)\right) \cup_{m=1,2 \ldots} E_{2 t_{m}}\left(-\frac{s_{m}}{2} e_{n}\right) .
$$

(see Figure 5.) Here $\pi / 10$ is an arbitrarily chosen small number such that the harmonic function associated with $W\left(\pi / 10,-e_{n}\right)$ decays strictly faster than quadratically. (Instead of $\pi / 10$ one may choose any angle $\theta_{0}<\theta_{n}$.)

Since $\partial \Omega_{0} \cap\left\{y_{n}=-\frac{s_{m}}{2}\right\}$ is a subset of a hyperplane, we may let $\beta_{n}=1$ in Lemma 3.2 and obtain

$$
h_{0}\left(-s_{m} e_{n}\right) \approx \frac{s_{m}}{t_{m}} h_{0}\left(-t_{m} e_{n}\right)>\frac{s_{m}^{2}}{t_{m}^{2+\delta}} h_{0}\left(-t_{m} e_{n}\right)
$$

Also since $t_{m+1}=s_{m} \cdot\left(\frac{s_{m}}{t_{m}}\right)^{A}$, if $A$ is sufficiently large and if $r<1$, then

$$
h_{0}\left(-r e_{n}\right) \leq r^{2+\epsilon} \text { for some } \epsilon>0 .
$$

Next let us consider a solution $v(x, t)$ of the heat equation in $\Omega_{0} \times[0, \infty)$ with initial data $v(x, 0) \in C^{\delta}\left(\mathbb{R}^{n}\right)$ satisfying $\Omega_{0}=\{v(x, 0)>0\}$ and $v\left(-r e_{n}, 0\right) \geq r^{\delta}$ for sufficiently small $r$. 


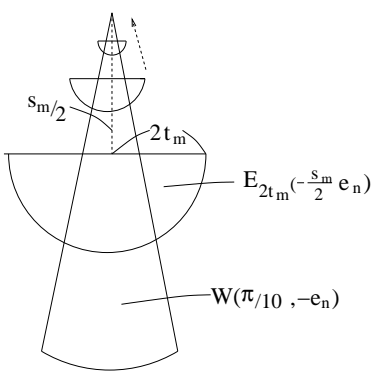

A counterexample for Theorem 1.8: constructing the initial domain

Figure 5 .

Due to Theorem 4.1,

$$
v\left(-r e_{n}, t\right) \geq C r^{\delta} \text { for } 0 \leq t \leq r^{2}, r<<1 .
$$

On the other hand by Lemma 4.3 there is $\epsilon>0$ such that $v-v^{1+\epsilon}$ is positive and superharmonic in $|x| \leq c_{0} t^{1 / 2}$ for a universal constant $c_{0}>0$. Hence by Lemma 4.4 there is a universal constant $C>0$ such that

$$
v\left(-s e_{n}, t\right) \geq C \frac{v\left(-t^{1 / 2} e_{n}, t\right)}{h\left(-t^{1 / 2} e_{n}, 0\right)} h\left(s e_{n}, 0\right) \text { for } s<c_{0} t^{1 / 2},
$$

By (5.1)-(5.2) we then obtain

$$
v\left(-s_{m} e_{n}, t\right) \geq C 2^{k_{0}} \frac{s_{m}^{2}}{t_{m}^{2}} \text {. for } t \in\left(t_{m}^{2}, 2 t_{m}^{2}\right)
$$

Now consider a viscosity solution $u$ of $(\mathrm{ST})$ with initial data $v(x, 0)$. By the maximum principle for the heat equation, $u$ is bigger than $v$. In particular one can replace $v$ by $u$ in (5.4).

Now let $\xi \in \Gamma_{0} \in\left\{x_{n}=-s\right\}$. Then by (5.4) and a barrier argument with radially symmetric subsolution of (ST) as in the proof of Lemma 4.5, we obtain that

$$
\operatorname{dist}\left(\xi, \Gamma_{2 t_{m}^{2}}(u)\right) \sim C \frac{u\left(-s_{m} e_{n}, t\right)}{s_{m}} \cdot t_{m}^{2} \geq C 2^{k_{0}} s_{m} .
$$


(5.5) and the fact that $t_{m}$ and $s_{m}$ is a decreasing sequence which converges to zero implies that for any small $t>0$ there exists $r>0$ such that

$$
\Gamma_{t}(u) \cap\left\{x \in \mathbb{R}^{n}:|x|<r\right\}=\varnothing .
$$

In other words there is no waiting time for $u$.

\section{Acknowledgement}

The authors thank the referee for pointing out the result of $[\mathrm{K}]$.

\section{References}

[A] N. D. Alikakos, On the pointwise behavior of the solutions of the porous medium equation as $t$ approaches zero or infinity, Nonlinear Anal. 9 (1985), 1095-1113.

[AC] D. G. Aronson and L.A. Caffarelli, The Initial Trace of a Solution of the Porous Medium Equation, Trans. Amer. Math. Soc. 280 (1983), no.1, 351-366.

[ACS] I. Athanasopoulos, L. Caffarelli and S. Salsa, Caloric functions in Lipschitz domains and the regularity of solutions to phase transition problems, Annals of Math., 143(1996), 413-434.

[C1] L. Caffarelli, A Harnack inequality approach to the regularity of free boundaries, Part I: Lipschitz free boundaries are $C^{1, \alpha}$, Rev. Mat. Iberoamericana 3 (1987), no. 2, 139-162

[D] B.E. Dahlberg, Harmonic functions in Lipschitz domains, Harmonic analysis in Euclidean spaces, Part 1, pp. 313-322, Proc. Sympos. Pure Math., XXXV, Part, Amer. Math. Soc., Providence, R.I., 1979

[Di] E. DiBenedetto, Chapter V. of Partial Differential Equations, Birkhäuser, 1995.

[EJ] C. M. Elliot and V. Janovsky, A variational inequality approach to Hele-Shaw flow with a moving boundary, Proc. Roy. Soc. Edinburgh. Sect. A 88 (1981), no.1-2, 93-107.

[ES] J. Escher, G. Simonett, Classical solutions of multidimensional HeleShaw models, SIAM J. Math. Anal 28 (1997), no.5, 1028-1047. 
[JK] D. S. Jerison and C. E. Kenig, Boundary behavior of Harmonic functions in Non-tangentially Accessible Domains, Advan. in Math. 46 (1982), 80-147.

[K] J.R. King, Development of singularities in some moving boundary problems, Euro. J. Appl. Math. 6,(1995) 491-507.

[Ki1] I. Kim, Uniqueness and Existence result of Hele-Shaw and Stefan problem, Arch. Rat. Mech. Anal, 168 (2003), 299-328.

[Ki2] I. Kim, Long time regularity of Hele-Shaw problem, submitted.

[KLV] J.R. King, A.A. Lacey and J.L. Vazquez, Persistence of corners in free boundaries in Hele-Shaw flow, Euro. J. Appl. Math., 6(1995), 455-490.

[Me] A. M. Meirmanov, The Stefan Problem, Walter de Gruyter, Berlin, 1992. MR 92m:35282

[Ru] L. I. Rubinstein, The Stefan Problem, Transl. Math. Monographs, vol.27, AMS, Providence, RI, 1971. MR 50:3837

[ST] P. G. Saffman and G.I. Taylor, The penetration of fluid into a porous medium Hele-Shaw cell containing a more viscous liquid, Proc. Royal Soc. London Ser. A 245 (1958),312-329. MR20:3697. 